\section{Modelagem matemática da evolução do fenótipo indutor de sincício na infecção HIV-1/AIDS}

\section{Mathematical modeling of the syncytium-inducing phenotype evolution in HIV-1/AIDS Pathogenesis}

\section{Resumo}

É proposta e discutida neste trabalho a modelagem matemática do processo de geração de variantes do vírus da imunodeficiência humana do tipo 1 que apresentam o fenótipo indutor de sincício durante as fases assintomática e AIDS clínica. Tais variantes podem fazer uso de CXCR 4 exclusivamente (variantes X4) ou não (variantes R5X4 ou dual-trópicas). A base experimental de Shankarappa et al. U Virol 1999; 73(2): 10489-502) é empregada como referência para derivação e calibração paramétrica de modelos fenomenológicos. Neste artigo, diferentes modelos matemáticos de evolução fenotípica do HIV-1, em termos da habilidade retroviral de induzir a formação de sincício, são propostos, testados e discutidos. Melhor aderência a resultados experimentais é verificada quando é considerada a reversibilidade entre fenótipos SI e NSI. Em adição, maior alinhamento à dinâmica in-vivo é observada se funções dependentes do tempo são admitidas para modelar a taxa de mutação entre variantes R5, R5X4 e X4 do HIV-1.

Palavras-chave: AIDS. Indutor de sincício. Modelagem matemática.

\author{
Marcel Joly',"II \\ José Maurício Pintol, 1 II \\ ' Departamento de Engenharia Química, Escola Politécnica da Universidade de \\ São Paulo. \\ " Petrobras Petróleo Brasileiro S.A. \\ III Othmer Department of Chemical and Biological Sciences and Engineering, \\ Polytechnic University, Six Metrotech Center, Brooklyn NY 11201, Estados \\ Unidos.
}

*Abreviações utilizadas neste artigo: AIDS: Acquired Immune Deficiency Syndrome; ACMK: autologous infected cell-mediated killing; AICD: Activation Induced Cell Death; APC: Antigen Presenting Cell; BBB: Blood-Brain Barrier; CNS: Central Nervous System (Compartment); CSF: cerebrospinal fluid; CTL: Cytotoxic T-Lymphocytes; ESI: (Time of) Emergence of Syncytium Inducing (HIV-1 Strains); FDC: Follicular Dendritic Cells; HAART: Highly Active Antiretroviral Therapy; HIV-1: Human Immunodeficiency Vírus Type-1; IL-2: Interleukin-2; LT: Lymphoid Tissues (Compartment); MHC: Major Histocompatibility Complex; NSI: Non Syncytium Inducing; PB: Peripheral Blood (Compartment); PBMC: Peripheral Blood Mononuclear Cells; SI: Syncytium Inducing.

Apoio: FAPESP (processo 99/09897-4) e Wechsler Award for Excellence

Correspondência: José Maurício Pinto, Praxair Inc., 39 Old Ridgebury Rd., Danbury CT, 06810, USA. 


\section{Abstract}

The mathematical modeling of the process of generation of HIV-1 strains that present the syncytium-inducing phenotype during the asymptomatic and clinical AIDS phases is proposed and discussed in this paper. These strains may utilize exclusively CXCR4 (X4 strains) or not (R5X4 strains or dualtropic). The experimental basis of Shankarappa et al. ( Virol 1999; 73(2): 10489-502,) is used as a reference for the generation and parameter estimation of the phenomenological models. In this study, different mathematical models for the phenotypical evolution of HIV-1 in terms of the retroviral ability to induce syncytium formation are proposed, tested and discussed. Better adherence to experimental results is verified when reversibility between the SI and NSI phenotypes is considered. Moreover, better alignment to in-vivo dynamics is observed if time-dependent functions are assumed to model the mutation rate among the R5, R5X4 and X4 strains of HIV-1.

Keywords: AIDS. Syncytium-inducing. Mathematical modeling.

\section{Manuscript presentation}

While the non-syncytium-inducing (NSI) $\rightarrow$ syncytium-inducing (SI) phenotypic evolution throughout disease progression is well documented (Figure 1), only in recent years comprehensive studies addressed the relationships among R5, R5X4 and X4 HIV-1 strains. In this paper, two questions are in the core of the discussion: a) although viruses originated in the late stages are characterized by the ability of using both CCR5 and CXCR4 coreceptors, does this fact reflect the prevalence of R5X4 or R5 + X4 strains? b) while the presence of SI R5X4 viruses at the final stages is well documented, its position within the viral phenotypic evolution is not clear; does it represent the last stage of the viral evolutionary change indeed corresponding to the fittest viruses or is it a simple intermediate during the $\mathrm{R} 5 \rightarrow \mathrm{X} 4$ transition, which may be not completed due to lack of available time (i.e., the death of the HIV-1 infected individual would occur first)?

Although the relevant genotypic similarity among env genes from several variants does not allow a formal dynamic analysis of the relationships among R5, R5X4 and X4 strains, one may conclude that:

- R5 strains persist to the emergence of CXCR4 using viruses;

- R5X4 viruses arise from the R5 lineage and

- X4 viruses can ultimately emerge from the R5X4 lineage, indeed indicating the intermediate condition of this phenotype.

Therefore, the phenotypic evolution of the envelope specificity may, in principle, follow a model that involves necessarily two irreversible transition stages: R5 $\rightarrow$ R5X $4 \rightarrow$ X4 (M1). However, this model may be unrealistic at least because it implies irreversible growth of the X4 phenotype without the typical peak in SI population (Figure 4a). On the other hand, regarding the presumed random nature associated to HIV-1 genomic mutation, a more realistic scenario would 
consider reversbility among phenotypes: $\mathrm{R} 5 \leftrightarrow \mathrm{R} 5 \mathrm{X} 4 \leftrightarrow \mathrm{X} 4$ (M2) .

Indeed, the relaxation of M1 into M2 regarding a time-dependent function for the mutation rate allows the derivation of a model that is able to predict a peak of SI population (Figure 4c). The agreement between these theoretical results and the predominance of R5X4 isolates in the late stages of the infection is observed.

\section{Introdução}

A infecção pelo vírus da imunodeficiência do tipo 1 (HIV-1) é caracterizada por um longo período assintomático de extensão variável que sucede a fase infecção primária e precede o estágio de AIDS clínica ${ }^{1}$.

Tal período de latência clínica, ainda hoje não compreendido em detalhes ${ }^{1-4}$, é caracterizado por processos biológicos complexos e altamente dinâmicos cujo resultado macroscópico traduz-se quantitativamente em populações celulares e viral quase-estacionárias ${ }^{5}$.

Diversos fatores inerentes ao hospedeiro e ao vírus têm sido identificados como potenciais determinantes da evolução da doença. Inerente ao hospedeiro, a diferença na qualidade e extensão da resposta imunológica entre indivíduos emerge como principal determinante. Neste sentido, o nadir virológico subsequente ao período de infecção primária, ou alternativamente, a resposta imune do hospedeiro durante esta fase, apresenta apreciável correlação com prognósticos de longevidade do mesmo.

Considerando que a variação genética do HIV-1 é a característica marcante desta infecção ${ }^{6}$, aspectos relacionados ao curso natural de evolução genotípica/fenotípica viral durante a fase assintomática têm sido amplamente investigados e associados à taxa de progressão da doenç $\mathrm{a}^{1,3,4,7,8}$. Pressões seletivas exercidas pela resposta imunológi$\mathrm{ca}^{9}$ associadas à infidelidade na transcrição reversa ${ }^{6,8,10}$ e à gama de células alvo disponível para infecção no hospedeiro ${ }^{10}$ são prováveis fatores de contribuição para a típica variabilidade genética do $\mathrm{HIV}-1^{8}$. A falta de uma atividade de leitura de prova (proofreading) ${ }^{10,11}$ ou atividade exonucleolítica ${ }^{12} \mathrm{da}$ enzima transcriptase reversa, competição darwiniana entre linhagens distintas ${ }^{9,10} \mathrm{e}$ recombinação $0^{11}$ complementam o conjunto de fatores predominantemente mencionados pela literatura como elementos motrizes de tal evolução genômica durante o curso da infecção.

Este artigo defere atenção sobre tradução matemática de evidências experimentais decorrentes de mutação viral que possuam, segundo a literatura, relevância para o delineamento da evolução da infecção subsequente ao clímax de virulência inicial.

Dentre os aspectos considerados, está a observação experimental relacionada à emergência de variantes do HIV-1 que utilizam o co-receptor CXCR4 para infecção celular. Tais variantes podem fazer uso de CXCR4 exclusivamente (variantes X4) ou não (variantes R5X4 ou dual-trópicas ${ }^{4}$ ). Considerando-se que estas variantes emergem tipicamente durante os estágios finais da infecção ${ }^{7,13}$, a derivação de tal modelagem revela-se importante para o estudo das fases assintomática e AIDS clínica, pois:

- as variantes X4 (e R5X4) apresentam espectro expandido de células alvo ${ }^{7,14}$, bem como forte tropismo a linfócitos $\mathrm{T} \mathrm{CD}^{+}$quiescentes $^{13}$ (cerca de $98 \%$ da população de linfócitos $\mathrm{T}^{5}$ ) ou recentemente ativados;

- como em Yamaguchi \& Gojobori ${ }^{6}$, os clones do HIV-1 são aqui divididos em 2 categorias: indutores de sincício (SI, syncytium inducing) e não indutores de sincício (NSI, non-syncytium inducing). Admite-se que variantes X4 são SI exclusivamente $^{2,7}$, enquanto as linhagens R5 são assumidas exclusivamente NSI. As linhagens R5X4, cuja infecção celular pode ser mediada por CCR5 e CXCR4 serão consideradas de fenótipo $\mathrm{SI}^{1,2,8}$ Isto é relevante pois as variantes SI apresentam cinética de replicação diferenciada $^{2,7}$;

- a quimioterapia anti-HIV-1 apresenta especificidade ao fenótipo viral ${ }^{14}$;

- as evidências acumuladas sugerem ser 
desejável a reversão da emergência de linhagens SI em terapias anti-HIV- ${ }^{14}$. De fato, o primeiro e o terceiro item motivam tal objetivo;

- viabiliza-se a avaliação computacional de hipóteses inerentes à derivação de variantes SI a partir de linhagens NSI em diferentes compartimentos anatômicos do hospedeiro ${ }^{9}$.

\section{Fundamentação}

A expressiva taxa de substituição de nucleotídeos nos genes do HIV- $1^{10}$ promove a formação de uma população viral heterogênea, porém constituída por indivíduos intimamente relacionados entre si geneticamente ${ }^{6}$. Dentre as diversas sequências geradas por sucessivos ciclos de replicação viral, algumas são eliminadas devido a desvantagens relacionadas ao seu maquinário reprodutivo, enquanto outras apreciam vantagens seletivas devido a novas características que facilitam seu escape à perseguição imunológica do hospedeiro ${ }^{6}$.

Os padrões evolutivos do genótipo e do fenótipo viral fortemente associados à progressão da doença caracterizam-se pela substituição parcial de linhagens NSI (R5) por variantes $\mathrm{SI}^{1,3,10}$ (R5X4; X4). Entretanto, os mecanismos biológicos de evolução do HIV-1 in vivo ainda não se encontram totalmente elucidados ${ }^{3}$. Neste estudo, as variações na sequência do gene $e n v$ são consideradas visando à avaliação da dinâmica de evolução do HIV-1, pois o gene env codifica a especificidade do co-receptor celular ao HIV-1 bem como seu fenótipo NSI ou $\mathrm{SI}^{1,3,6-8}$. Além disso, o gene env constitui um importante elemento de reconhecimento por parte dos linfócitos $\mathrm{T} \mathrm{CD8}{ }^{+}$citotóxicos $^{6}$ (CTLs). Portanto, mutações ocorridas no mesmo podem ter relevância no que concerne à eficiência da resposta imune do hospedeiro.

Em Shankarappa et al. (1999), um dos desenvolvimentos mais abrangentes publicados nesta direção, a evolução do HIV-1 ao longo do curso da infecção é analisada sob 3 aspectos distintos, sendo 2 deles genotípi- cos (em relação à região C2-V5 do gene env) e um fenotípico:

- A divergência: grau de variabilidade genotípica das linhagens presentes no hospedeiro em um dado instante em relação à sequência da variante inicialmente presente no hospedeiro;

- A diversidade: grau de variabilidade genotípica da população viral em relação à linhagem predominante para um dado instante;

- A representatividade do fenótipo SI: fração da população viral com fenótipo SI em um dado instante.

Como em Shankarappa et al. ${ }^{1}$, a fase assintomática é aqui avaliada em 3 estágios. No primeiro, divergência e diversidade sofrem incrementos lineares e semelhantes, da ordem de $1 \%$ ao ano. O início do segundo estágio é caracterizado por estabilização, ou mesmo decréscimo na diversidade enquanto a população viral continua a divergir da variante inicial na mesma razão de $1 \%$. Ultimamente, a expansão na divergência também se estabiliza, ou se contrai, denotando o início do terceiro estágio, no qual a diversidade sofre redução. As variantes SI estão inicialmente ausentes, emergendo pouco antes do pico de diversidade (este, em média, de 4 a 9 anos após seroconversão [Sharankappa et al. ${ }^{1}$ ]) e apresentam pico populacional (em proporção superior a $80 \%$ ) fortemente associado ao pico de divergência (Figura 1). Dada a elevada taxa de replicação viral ${ }^{15}$ e o pequeno número de substituições no gene $e n v$ necessárias para alterar o fenótipo viral ${ }^{8}$, não está clara a razão pela qual a emergência de variantes SI é um evento que tipicamente ocorre anos após a seroconversão ${ }^{14}$.

Embora seja função do indivíduo, Shankarappa et al. ${ }^{1}$ determinaram que se o instante da emergência do fenótipo SI for tomado como zero, o pico de diversidade ocorrerá, em média, após 0,3 anos, o pico populacional de linhagens SI ocorrerá após 1,5 anos, a estabilização da divergência após 2,2 anos e a definição das condições clínicas de AIDS após 2,9 anos. 


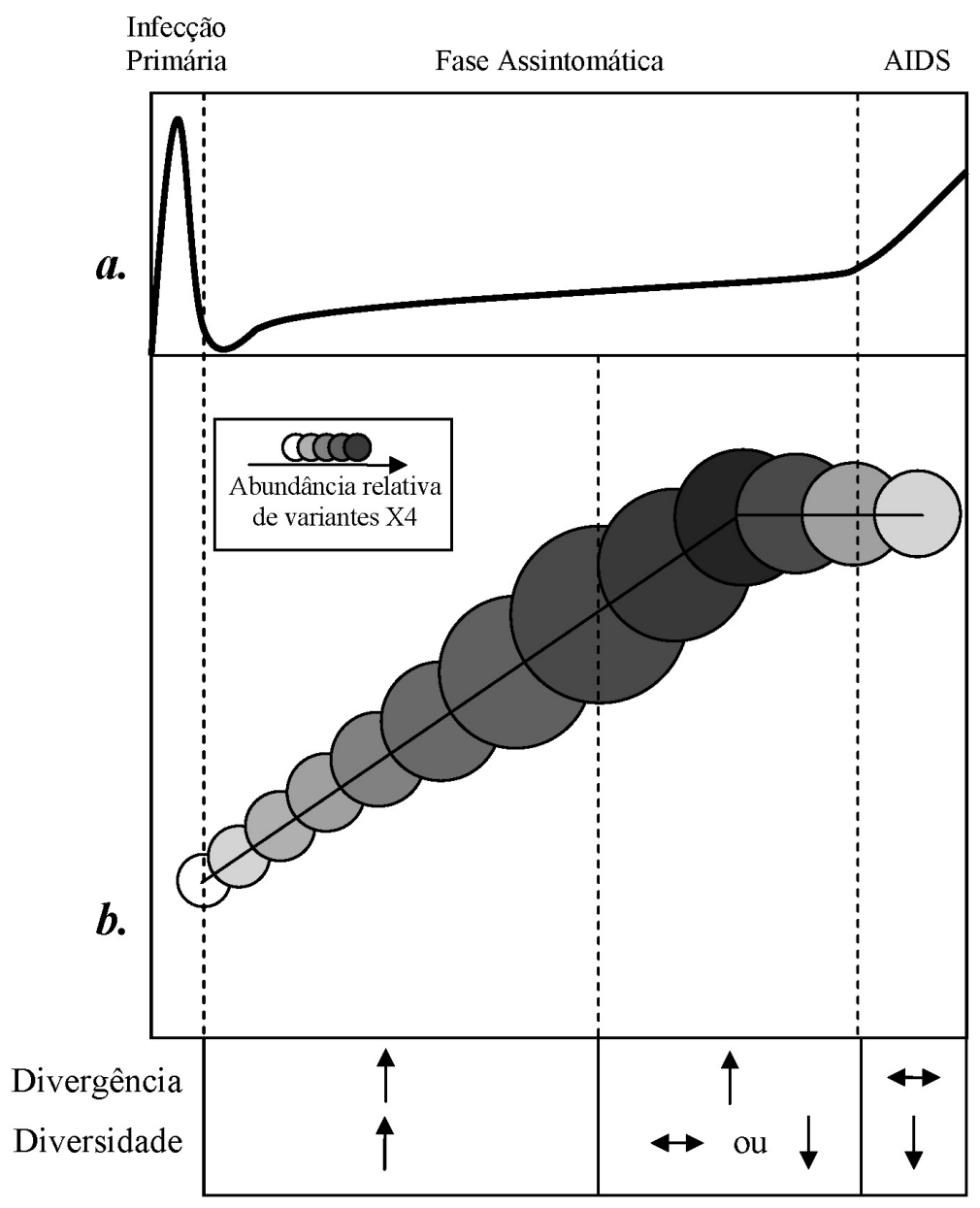

Figura 1 - Padrões típicos de evolução do HIV-1 ao longo da progressão da doença (extraído de Shankarappa et al. $\left.{ }^{1}\right)$. $\boldsymbol{a}$. Fases clínicas da infecção HIV-1/AIDS e padrões típicos de carga viral e concentração de linfócitos $\mathrm{TCD}^{+}$. $\boldsymbol{b}$. Padrões da evolução de sequência viral dentro do período assintomático: o diâmetro dos círculos denota a amplitude da diversidade após seroconversão; a posição vertical dos círculos denota a extensão da divergência; a intensidade do sombreamento informa a representatividade do fenótipo SI (ver legenda na figura). Legenda: $\uparrow=$ elevação; $\downarrow=$ decréscimo; $\leftrightarrow$ = estabilização.

Figure 1 - Typical behavior of the HIV-1 infection throughout disease progression (from Shankarappa et al. ${ }^{1}$ ). a. Clinical phases and qualitative profiles for typical viral load and CD4+ T-lymphocyte count. $\boldsymbol{b}$. Typical viral phenotypic evolution associated with the asymptomatic period: circle diameters denote the amplitude of the HIV1 population diversity after seroconversion whereas their vertical positions indicate the corresponding magnitude of the viral genotypic divergence. SI presence is represented by the circle shading intensity. Legend: $\uparrow=$ increase; $\downarrow$ $=$ decrease $\leftrightarrow$ = stability.

\section{Hipóteses biológicas}

\section{Dinâmica evolutiva de variantes refratárias à ação citotóxica}

O reconhecimento de CTLs infectadas é dependente de eventos intracelulares complexos. Tais eventos envolvem a degradação de proteínas virais em fragmentos (peptídeos) que são posteriormente associados a moléculas MHC da classe I sobre a su- perfície da célula infectada ${ }^{16}$, constituindo epítopes para CTLs específicas ao HIV-1. Há crescente evidência relacionada ao papel crítico que tal resposta citotóxica exerce sobre o controle da replicação viral, bem como sobre a progressão da doença. Por outro lado, mutações naturais em proteínas regulatórias e acessórias do HIV-1 que ocorrem durante a evolução da doença implicam alterações nos epítopes sintetizados sobre a célula infectada. Tais divergências geno- 
típicas em relação ao padrão inicial (para o qual a resposta citotóxica primeiramente elaborada é específica) podem reduzir ou eliminar o reconhecimento da célula infectada pela CTL.

Não se verifica consenso quanto ao epítope preponderante para a típica redução da resposta citotóxica das CTLs durante as fases finais da infecção. Enquanto uns sugerem seleção positiva para o epítope env e não para gag, outros propõem especificidade predominante ao epítope gag durante a fase assintomática da infecção. No entanto, a existência de especificidade citotóxica a env é aparentemente consensual ${ }^{6}$, sendo inclusive mencionada como o principal alvo da ação imune do hospedei$\mathrm{ro}^{12}$. De fato, inequívoca demonstração de mutações refratárias à ação citotóxica que emergem ao longo da fase assintomática especificamente em relação ao epítope $e n v$ tem sido associada à expansão viral e à inabilidade do sistema imune em prevenir a eclosão da AIDS clínica ${ }^{6}$. Neste sentido, é considerado que:

- a eficiência da ação citotóxica das CTLs segue função exclusiva da variabilidade do gene env, em particular da região C2$\mathrm{V}^{1}$;

- a evolução do genótipo da região C2V5 reportada em Shankarappa et al. ${ }^{1}$ é representativa da variabilidade de todas as regiões do gene $e n v$;

- toda mutação considerada em Shankarappa et al. ${ }^{1}$ implica seleção positiva da variante gerada. A motivação para tanto reside no fato de que as variantes menos aptas tendem a ser (rapidamente) extintas ao longo do processo evolutivo ${ }^{10}$.

\section{Dinâmica evolutiva do fenótipo viral indutor de sincício (SI)}

Embora a emergência de linhagens SI seja frequentemente associada a aproximadamente $50 \%$ dos casos de infecção pelo HIV- $1^{9,14}$, a confiabilidade de tal informação tem sido colocada à prova ${ }^{1}$ porque em geral é obtida via ensaios fenotípicos, mais susceptíveis a falhas do que os testes genotípicos. Em adição, a presença destas variantes virais pode ser transiente durante o curso da infecção. Portanto, estimativas equivocadas sobre a proporção de indivíduos que as hospedam ao longo da progressão da doença podem ser produzidas ${ }^{1}$.

Em alinhamento a Shankarappa et al. ${ }^{1}$, será assumido que a taxa de emergência de variantes SI em indivíduos infectados pelo HIV-1 seja substancialmente superior a 50\%. É admitido que tal evento ocorre em $100 \%$ dos casos. Embora conservativa (variantes SI são tipicamente mais patogênicas), tal hipótese é necessária para viabilizar o caráter determinístico do modelo matemático proposto.

Negligenciando possíveis influências (como competição darwiniana) de linhagens R5 NSI sobre a evolução populacional de variantes SI e desprezando o período de tempo entre a infecção inicial e a seroconversão, a evolução populacional de linhagens que usam o co-receptor CXCR4 é modelada parametricamente em $t$ (tempo em anos). A emergência e o pico de representação de variantes SI dentro da população viral são, em média, detectados aproximadamente em $(\Xi-0,3)$ e $(\Xi+1,2)$ anos, respectivamente ${ }^{1}$. Por simplificação, a taxa de crescimento da população SI entre ambos os eventos é considerada linear com pico de representatividade SI definido pelo parâmetro $\Omega \%(\Omega \in \Re \mid 80 \%<\Omega<100 \%)^{1}$. Subsequente a esta condição de pico, assume-se contração linear da população SI, em $\tau \%$ ao ano $(\tau \in \mathfrak{R} \mid \tau \geq 0)$.

A fração de variantes SI $\left(F_{S I}(t), 0<F_{S I}(t)\right.$ $<1$, onde $t=$ tempo em anos após seroconversão) dentro da população viral do hospedeiro fica, portanto, modelada como em Shankarappa et al. ${ }^{1}$.

Alternativamente a este modelo, podem ser também utilizadas funções de aproximação para representação populacional da linhagem SI em progressores típicos. Como ilustração desta abordagem, os perfis observados para os indivíduos 1, 2 e 3 (progressores típicos) de Shankarappa et al. ${ }^{1}$ estão aqui reproduzidos na Figura 2 (i-iii). Aproximações podem ser estimadas com 
$F_{S I}(t)=\left\{\begin{array}{l}0, \text { para } \quad(\Xi-0,3)>t>\left(\frac{\Omega}{\tau}\right)+(\Xi+1,2) \\ \left(\frac{\Omega}{1,5}\right) \mathrm{t}, \text { para }(\Xi-0,3) \leq t \leq(\Xi+1,2) \\ \Omega-\tau(t-(\Xi+1,2)), \text { para }(\Xi+1,2)<t \leq\left(\frac{\Omega}{\tau}\right)+(\Xi+1,2)\end{array}\right.$

$F_{S I}(t)=\frac{\alpha}{\beta_{1}+\delta_{1} e^{-\left(t-t_{1}\right) \Delta T_{1}}}-\frac{\alpha}{\beta_{2}+\delta_{2} e^{-\left(t-t_{2}\right) \Delta T_{2}}} \quad t>0$

base em (2), como mostra a Figura 2 (iv-vi). Outras funções poderiam ser igualmente empregadas.

Visando definir relações matemáticas entre a taxa de geração de variantes SI e a correspondente fração numérica destas dentro da população viral do hospedeiro, é preciso considerar que:

- a população viral (assim como a de linfócitos $\mathrm{T} \mathrm{CD}^{+}$) é vertiginosamente substituída ${ }^{15}$; a vida média da partícula viral ( $\cong 6$ horas $^{15}$ ), bem como o ciclo replicativo viral $\left(\cong 2,6\right.$ dias $\left.^{17}\right)$, são desprezíveis perante o horizonte de tempo da fase assintomática (escala de anos).

- a ação anti-HIV-1 devida ao sistema imune é aproximadamente equivalente sobre ambas linhagens R5 e X4/R5X4. Embora a secreção de fatores solúveis tenham sido associados à inibição preferencial da infecção celular por linhagens NSI (R5) do HIV-1, a principal

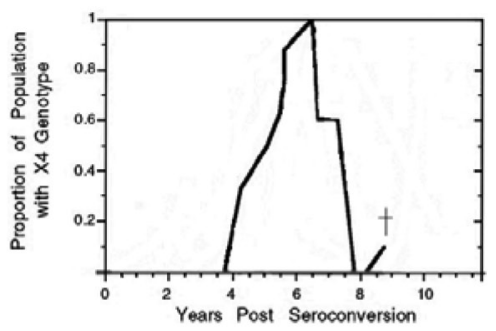

(i)

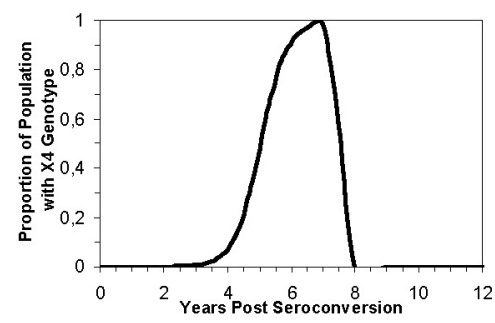

(iv)

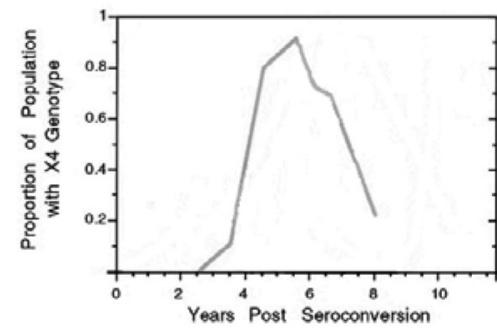

(ii)

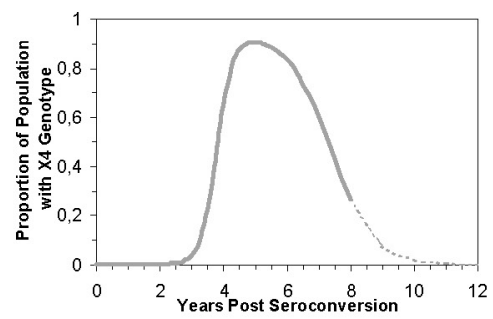

(v)

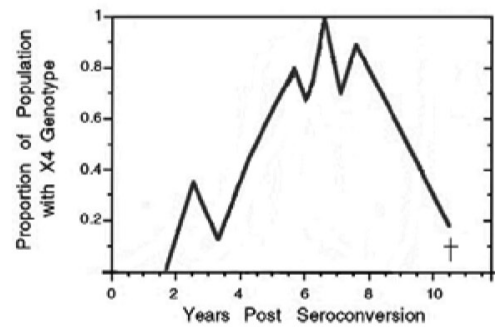

(iii)

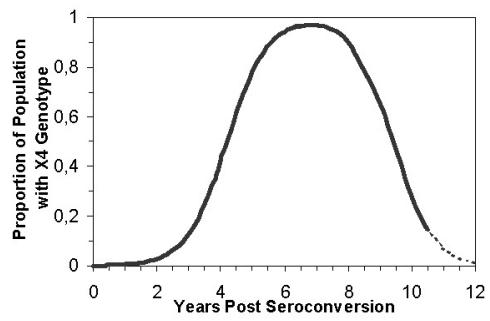

(vi)

Figura 2 - Perfis experimentais ${ }^{1}$ (i-iii) e aproximados (iv-vi) por função da forma da eq. (2) da fração populacional de variantes com genótipo SI. Dados para os indivíduos (progressores típicos) 1 (i;iv), 2 (ii;v) e 3 (iii;vi) de Shankarappa et al. ${ }^{1}$. Marcador (†) em (i; iii) denota interrupção experimental devido à morte do indivíduo. Valores dos parâmetros ajustados para os indivíduos 1,2 e $3:\left(\alpha, \beta_{1^{\prime}} \beta_{2^{\prime}} \delta_{1^{\prime}}, \delta_{2^{\prime}} t_{1^{\prime}}\right.$ $\left.t_{2^{\prime}} \Delta T_{1^{\prime}} \Delta T_{2}\right)=[(1 \%, 1,005,1,005,1,1,1,4,5,0$ anos, 7,5 anos, 2,5 anos, 15 anos), (1\%, 1,070, 1,070, 1,0, 1,4, 3,9 anos, 7,2 anos, 3,8 anos, 1,5 anos); (1\%, 1,000, 1,000, 1,9, 1,9, 3,8 anos, 9,0 anos, 1,6 anos, 1,6 anos)].

Figure 2 - Experimental ( $(i$-iii) and approximated (iv-vi) profiles by a function of the form of eq. (2) of the population fraction of strains with the SI phenotype. Data for individuals (typical progressors) 1 (i;iv), 2 (ii; v) and 3 (iii;vi) from Shankarappa et al. ${ }^{1}$. Marker ( + ) in (i; iii) denotes the experimental interruption due to an individual's death. Values of the adjusted parameters for individuals 1,2 and $3:\left(\alpha, \beta_{1^{\prime}} \beta_{2^{\prime}} \delta_{1^{\prime}} \delta_{2^{\prime}} t_{1^{\prime}} t_{2^{\prime}} \Delta T_{1^{\prime}}\right.$ $\left.\Delta T_{2}\right)=[(1 \%, 1.005,1.005,1.1,1.4,5.0$ years, 7.5 years, 2.5 years, 15 years $),(1 \%, 1.070,1.070,1.0,1.4,3.9$ years, 7.2 years, 3.8 years, 1.5 years $) ;(1 \%$, $1.000,1.000,1.9,1.9,3.8$ years, 9.0 years, 1.6 years, 1.6 years $)]$. 
ação antiviral devida a mecanismos imunológicos do hospedeiro (atividade citotóxica das CTLs) é independente do envelope viral, agindo, portanto, sobre ambas as variantes do HIV-1;

- o reservatório latente de HIV-1 em humanos é constituído principalmente por células latentemente infectadas com linhagens R5 NSI do HIV- ${ }^{14}$.

Logo, cessada a geração de variantes SI, pode ser esperado rápido declínio da população viral com fenótipo SI.

\section{Evolução da especificidade viral a CCR5 e CXCR4}

Embora a ligação da glicoproteína viral gp120 (produzido pelo gene $e n v$ ) ao receptor CD4 celular seja virtualmente universal entre variantes do HIV-1, constata-se sensível variação na interação vírus-célula quanto à especificidade do envelope ao tipo de coreceptor celular ${ }^{8}$. Pressões seletivas e aspectos inerentes ao ciclo de replicação do HIV-1 são prováveis fatores de contribuição para a diversificação genética do gene env durante o curso da infecção ${ }^{8}$. Tal diversificação pode, como resultado, implicar a alteração do co-receptor utilizado para infecção celular. Embora uma ampla gama de co-receptores ao HIV-1 tenham sido identificada ${ }^{13}$, este estudo considera CCR5 e CXCR4 os principais co-receptores ao HIV- $1^{13,14}$, posto que todas linhagens do vírus são aptas ao uso de ao menos um deles ${ }^{8}$.

Sequências do gene $e n v$ do HIV-1 presente em fases iniciais da infecção revelam uso exclusivo do co-receptor CCR5. Assumidas exclusivamente NSI, estas linhagens evoluem tipicamente para variantes que incluem o uso do co-receptor CXCR $4^{3,8} \mathrm{e}$ apresentam fenótipo SI. Embora a evolução fenotípica NSI $\rightarrow$ SI durante o curso da doença esteja bem estabelecida ${ }^{1-4,8,9}$, tem sido publicado um número limitado de pesquisas abrangentes envolvendo as relações entre variantes R5, R5X4 eX4. Neste contexto, é oportuna a análise de duas questões:

- embora as variantes virais presentes nas fases finais da doença possuam habilidade de utilizar ambos co-receptores (CCR5 e CXCR4), isto revela domínio das linhagens R5X4 ou R5 + X4?

- embora a presença de variantes R5X4 nos estágios finais da doença esteja bem documentada ${ }^{3}$, a posição desta na evolução viral não está clara. As variantes R5X4 podem representar o estágio final da evolução ${ }^{3}$ (variante mais apta) ou um intermediário durante a transição $\mathrm{R} 5 \rightarrow \mathrm{X} 4^{4}$, transição esta por vezes não observada devido à provável falta de tempo para que a evolução R5X4 $\rightarrow$ X4 seja consumada ${ }^{3}$ (a morte do hospedeiro ocorreria primeiro).

A similaridade genotípica entre genes $e n v$ de diversas variantes não permite definir, do ponto de vista temporal, a relação entre as linhagens R5, R5X4 e X4 in vitro ${ }^{3}$. Por outro lado, está bem estabelecido que as variantes R5 persistem à emergência de linhagens que utilizam o co-receptor CXCR4 $4^{9,14}$ e que as variantes R5X4 emergem a partir da linhagem R5 ${ }^{3}$. Não obstante, as variantes restritas ao uso de CXCR4 podem ultimamente emergir da população R5X4, indicando que estas últimas podem representar um intermediário evolucionário entre linhagens R5 e X48.

Com base nestas evidências, é inicialmente admitido que a evolução da especificidade do envelope ao tipo de co-receptor é unidirecional e necessariamente envolve dois estágios de transição (estágios $I$ e $I I$ ), como em (M1). Os tempos de meia-vida do HIV-1 livre e do ciclo intracelular de replicação viral são desprezíveis, ou seja, é assumido que a geração, replicação e morte da partícula viral ocorrem instantaneamente.

$R 5 \stackrel{I}{\rightarrow} R 5 X 4 \stackrel{I I}{\rightarrow} X 4$

Sob a hipótese de que toda partícula viral é viável e gera descendente único, admite-se estabilidade populacional do agente infeccioso (hipótese razoável para a fase assintomática) como em (3): 
$\gamma_{R 5 \rightarrow R 5}+\gamma_{R 5 \rightarrow R 5 X 4}-\delta_{R 5}=0$

$\gamma_{R 5 X 4 \rightarrow R 5 X 4}+\gamma_{R 5 X 4 \rightarrow X 4}-\delta_{R 5 X 4}=0$

$\gamma_{X 4 \rightarrow X 4}-\delta_{X 4}=0$

com:

$\delta_{R 5}=\delta_{R 5 X 4}=\delta_{X 4}=\delta=1$

$0 \leq \gamma_{a \rightarrow b} \leq 1 \quad(\mathrm{a}, \mathrm{b})$ e $E \mid E=\{(R 5, R 5),(R 5, R 5 X 4)$, $(R 5 X 4, R 5 X 4),(R 5 X 4, X 4),(X 4, X 4)\}$

onde $\gamma_{a \rightarrow b}$ denota a fração normalizada de geração (por ciclo de replicação) de variantes descendentes $b$ a partir de ascendente $a$ (mutação $a \rightarrow b$, se $a \neq b$ ).

Sob as hipóteses anteriores, a formalização matemática de (M1) resulta em (4), onde $V_{a}(t)$ para $a=R 5, R 5 X 4, X 4$ denota a fração normalizada da população $a$ no instante $t$.
O modelo biológico associado a (4) é apresentado na Figura 3a. A estimativa dos parâmetros $\gamma_{a \rightarrow b},(a, b) \in E$ foi realizada sobre a base dos resultados experimentais de Shankarappa et al. ${ }^{1}$. Segundo este procedimento, efetua-se o ajuste ótimo de (4) em relação a (1) (ou (2)) apenas para o período em que esta prediz $\left(d F_{S I}(t) / d t\right) \geq 0$, utilizando-se, para tanto, um objetivo tal qual (5) formulado para ajuste de (4) ao período monotonicamente crescente de (1) (ou de (2)), com $F_{S I}(t)$ e $\Xi$ definidos em (1) (ou (2)). A estimativa de $\gamma_{a \rightarrow b},(a, b) \in E$, segundo o procedimento descrito acima consiste na solução de um problema de otimização dinâmica não linear. Através da implementação computacional do modelo de otimização no ambiente GAMS ${ }^{18}$, sua solução foi produzida via método do gradiente reduzido generalizado (solver $\mathrm{CONOPT} 2^{19}$ ).

$$
\begin{aligned}
& \frac{d V_{R 5}(t)}{d t}=V_{R 5}(t) \cdot \gamma_{R 5 \rightarrow R 5}-V_{R 5}(t) \cdot \delta_{R 5} \\
& \frac{d V_{R 5 X 4}(t)}{d t}=V_{R 5}(t) \cdot \gamma_{R 5 \rightarrow R 5 X 4}+V_{R 5 X 4}(t) \cdot \gamma_{R 5 X 4 \rightarrow R 5 X 4}-V_{R 5 X 4}(t) \cdot \delta_{R 5 X 4} \\
& \frac{d V_{X 4}(t)}{d t}=V_{R 5 X 4}(t) \cdot \gamma_{R 5 X 4 \rightarrow X 4}+V_{X 4}(t) \cdot \gamma_{X 4 \rightarrow X 4}-V_{X 4}(t) \cdot \delta_{X 4} \\
& V_{R 5}(t)+V_{R 5 X 4}(t)+V_{X 4}(t)=1
\end{aligned}
$$$$
\operatorname{Min}_{\substack{a \rightarrow b \\(a, b) \in E}} \quad \int_{\Xi-0,3}^{\Xi+1,2}\left[\mathrm{~F}_{\mathrm{SI}}(t)-\left(V_{R 5 X 4}(t)+V_{X 4}(t)\right)\right]^{2} d t
$$

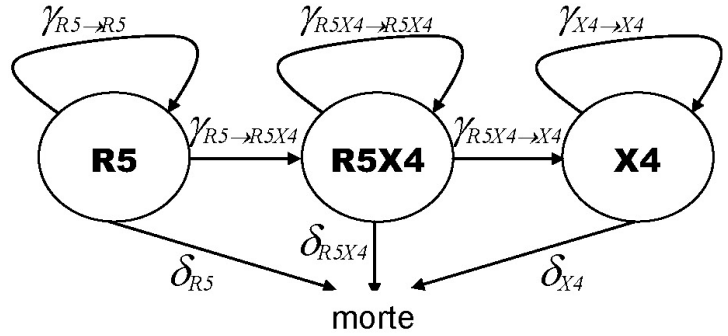

(a)

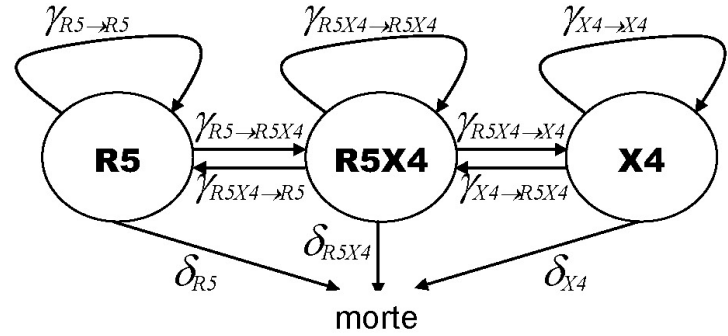

(b)

Figura 3 (a) Representação biológica inerente ao modelo (3). (b) Representação biológica inerente ao modelo (7). Condições iniciais: $V_{R 5}(t=0)=1$ (inicialmente, a população viral é homogênea e apresenta fenótipo $\left.R 5\right), V_{R 5 \times 4}(t=0)=0$ e $V_{\chi 4}(t=0)=0$.

Figure 3 (a) Biological representation related to the model (3). (b) Biological representation inherent to model (7). Initial conditions: $V_{R 5}(t=0)=$ 1 (initially, the viral population is homogeneous and has the $R 5$ phenotype), $V_{R 5 X 4}(t=0)=0$ and $V_{X 4}(t=0)=0$ 
Resultados típicos para a dinâmica evolutiva das linhagens R5, R5X4 e X4 no hospedeiro estão apresentados na Figura 4a.

O comportamento monotônico do modelo associado à expansão irreversível da fração populacional SI impede a predição de um pico de representação de linhagens SI, o que é tipicamente observado in-vivo 18 meses após a eclosão de variantes SI no hospedeiro ${ }^{1}$.

Claramente, dado o presumido caráter randômico associado à mutação genômica do HIV-1, uma hipótese mais realística acerca da dinâmica evolutiva postulada em (M1) poderia admitir reversibilidade nos estágios I e II, como em (M2):

$$
R 5 \stackrel{I}{\leftrightarrow} R 5 X 4 \stackrel{I I}{\leftrightarrow} X 4
$$

A relaxação de (M1) em (M2) permite reformular (3-4) como em (6).

O modelo biológico associado a (6) é apresentado na Figura 3b. Para o caso não trivial $\left(\gamma_{R 5 \rightarrow R 5 X 4}=0\right)$, o sistema (6) fica caracterizado pelo estado estacionário definido em (7). Tal condição estacionária, claramente distinta daquela anterior, encontra-se ilustrada na Figura 4b para um horizonte hipotético de 40 anos após a emergência de variantes SI.

A flexibilização de (M1) em (M2) associada à relaxação de $\gamma_{a \rightarrow b^{\prime}}(a, b) \in E^{R}$, em uma função contínua $\gamma_{a \rightarrow b}(t),(a, b) \in E^{R}$ definida para todo $t \geq 0$, viabiliza a derivação de um modelo apto à predição de um pico populacional da linhagem SI no hospedeiro. Admitindo-se taxas de mutação dinâmicas em (6), a evolução da população SI fica descrita por (8) e a condição (7) é reescrito como (9).

$$
\begin{aligned}
& \frac{d V_{R 5}(t)}{d t}=V_{R 5}(t) \cdot \gamma_{R 5 \rightarrow R 5}+V_{R 5 X 4}(t) \cdot \gamma_{R 5 X 4 \rightarrow R 5}-V_{R 5}(t) \cdot \delta_{R 5} \\
& \frac{d V_{R 5 X 4}(t)}{d t}=V_{R 5}(t) \cdot \gamma_{R 5 \rightarrow R 5 X 4}+V_{R 5 X 4}(t) \cdot \gamma_{R 5 X 4 \rightarrow R 5 X 4}+V_{X 4}(t) \cdot \gamma_{X 4 \rightarrow R 5 X 4}-V_{R 5 X 4}(t) \cdot \delta_{R 5 X 4} \\
& \frac{d V_{X 4}(t)}{d t}=V_{R 5 X 4}(t) \cdot \gamma_{R 5 X 4 \rightarrow X 4}+V_{X 4}(t) \cdot \gamma_{X 4 \rightarrow X 4}-V_{X 4}(t) \cdot \delta_{X 4} \\
& \gamma_{R 5 \rightarrow R 5}+\gamma_{R 5 \rightarrow R 5 X 4}-\delta=0 \\
& \gamma_{R 5 X 4 \rightarrow R 5}+\gamma_{R 5 X 4 \rightarrow R 5 X 4}+\gamma_{R 5 X 4 \rightarrow X 4}-\delta=0 \\
& \gamma_{X 4 \rightarrow R 5 X 4}+\gamma_{X 4 \rightarrow X 4}-\delta=0
\end{aligned}
$$

$$
\begin{aligned}
& V_{R 5}^{s s}=\frac{\gamma_{R 5 X 4 \rightarrow R 5} \cdot \gamma_{X 4 \rightarrow R 5 X 4}}{\gamma_{R 5 \rightarrow R 5 X 4} \cdot \gamma_{X 4 \rightarrow R 5 X 4}+\gamma_{R 5 X 4 \rightarrow R 5} \cdot \gamma_{X 4 \rightarrow R 5 X 4}+\gamma_{R 5 X 4 \rightarrow X 4} \cdot \gamma_{R 5 \rightarrow R 5 X 4}} \\
& V_{R 5 X 4}^{s s}=\frac{\gamma_{R 5 \rightarrow R 5 X 4} \cdot \gamma_{X 4 \rightarrow R 5 X 4}}{\gamma_{R 5 \rightarrow R 5 X 4} \cdot \gamma_{X 4 \rightarrow R 5 X 4}+\gamma_{R 5 X 4 \rightarrow R 5} \cdot \gamma_{X 4 \rightarrow R 5 X 4}+\gamma_{R 5 X 4 \rightarrow X 4} \cdot \gamma_{R 5 \rightarrow R 5 X 4}} \\
& V_{X 4}^{s s}=\frac{\gamma_{R 5 \rightarrow R 5 X 4} \cdot \gamma_{R 5 X 4 \rightarrow X 4}}{\gamma_{R 5 \rightarrow R 5 X 4} \cdot \gamma_{X 4 \rightarrow R 5 X 4}+\gamma_{R 5 X 4 \rightarrow R 5} \cdot \gamma_{X 4 \rightarrow R 5 X 4}+\gamma_{R 5 X 4 \rightarrow X 4} \cdot \gamma_{R 5 \rightarrow R 5 X 4}}
\end{aligned}
$$

$\frac{d F_{S I}(t)}{d t}=V_{R 5}(t) \cdot \gamma_{R 5 \rightarrow R 5 X 4}(t)-V_{R 5 X 4}(t) \cdot \gamma_{R 5 X 4 \rightarrow R 5}(t)$

$$
\begin{aligned}
& V_{R 5}^{s s}=\frac{\gamma_{R 5 X 4 \rightarrow R 5}(t) \cdot \gamma_{X 4 \rightarrow R 5 X 4}(t)}{\gamma_{R 5 \rightarrow R 5 X 4}(t) \cdot \gamma_{X 4 \rightarrow R 5 X 4}(t)+\gamma_{R 5 X 4 \rightarrow R 5}(t) \cdot \gamma_{X 4 \rightarrow R 5 X 4}(t)+\gamma_{R 5 X 4 \rightarrow X 4}(t) \cdot \gamma_{R 5 \rightarrow R 5 X 4}(t)} \\
& V_{R 5 X 4}^{s s}=\frac{\gamma_{R 5 \rightarrow R 5 X 4}(t) \cdot \gamma_{X 4 \rightarrow R 5 X 4}}{\gamma_{R 5 \rightarrow R 5 X 4}(t) \cdot \gamma_{X 4 \rightarrow R 5 X 4}(t)+\gamma_{R 5 X 4 \rightarrow R 5}(t) \cdot \gamma_{X 4 \rightarrow R 5 X 4}(t)+\gamma_{R 5 X 4 \rightarrow X 4}(t) \cdot \gamma_{R 5 \rightarrow R 5 X 4}(t)} \\
& V_{X 4}^{s s}=\frac{\gamma_{R 5 \rightarrow R 5 X 4}(t) \cdot \gamma_{R 5 X 4 \rightarrow X 4}(t)}{\gamma_{R 5 \rightarrow R 5 X 4}(t) \cdot \gamma_{X 4 \rightarrow R 5 X 4}(t)+\gamma_{R 5 X 4 \rightarrow R 5}(t) \cdot \gamma_{X 4 \rightarrow R 5 X 4}(t)+\gamma_{R 5 X 4 \rightarrow X 4}(t) \cdot \gamma_{R 5 \rightarrow R 5 X 4}(t)}
\end{aligned}
$$




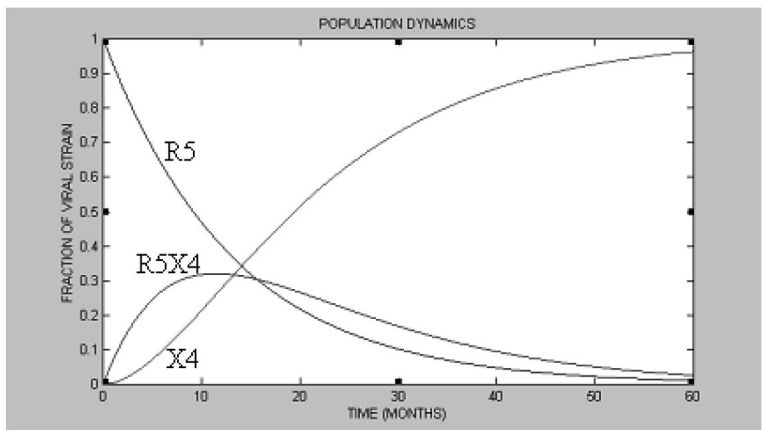

(a)

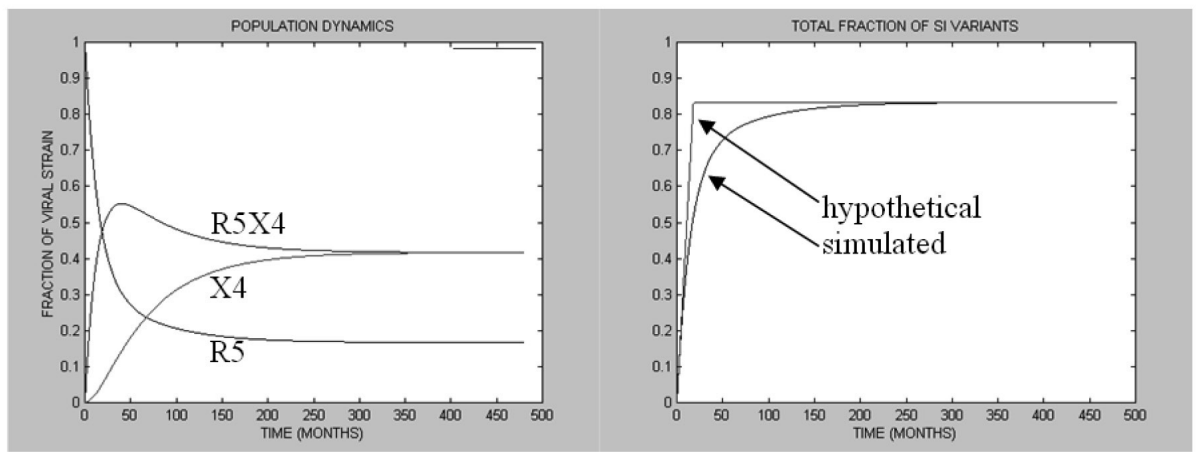

(b)

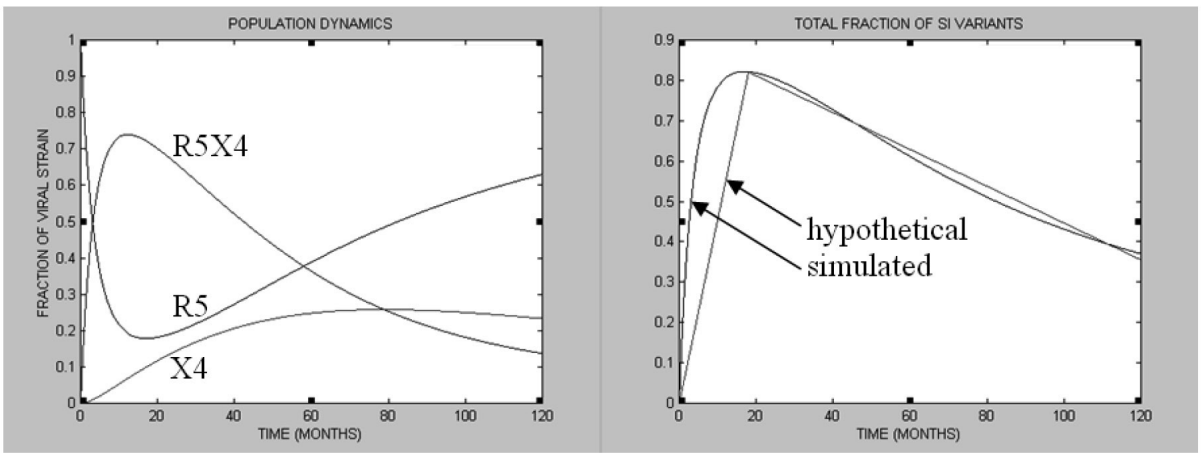

(c)

Figura 4 (a) Dinâmica populacional das variantes R5, R5X4 e X4 produzida por simulação de (3-4) para valores ajustados de $\gamma_{a \rightarrow b^{\prime}}(a, b)$ $\in \mathrm{E}$ para $\Omega=80 \%$; (b) (Esquerda) Perfis da dinâmica populacional de variantes do HIV-1 subsequente à emergência de linhagens $\mathrm{SI}$ gerados por simulação de (6). Aqui: $\gamma_{R 5 \rightarrow R 5}=0,95 ; \gamma_{R 5 \rightarrow R 5 X 4}=0,05 ; \gamma_{R 5 X 4 \rightarrow R 5}=0,02 ; \gamma_{R 5 X 4 \rightarrow R 5 X 4}=0,97 ; \gamma_{R 5 X 4 \rightarrow X 4}=0,01 ; \gamma_{X 4 \rightarrow R 5 X 4}=0,01 ; \gamma_{X 4 \rightarrow X 4}=0,99$.. (Direita) Evolução da população SI (R5X4+X4). Em preto, perfil SI correspondente à simulação de (6); em vermelho, correspondente a (1) para $\Omega$ $=83,33 \%$ e $\tau \rightarrow 0 \%$; (c) (Esquerda) Dinâmica populacional de variantes R5, R5X4 e X4 após eclosão do fenótipo SI gerada por simulação de (6) com taxas dinâmicas de mutação. Aqui, $\gamma_{R 5 \rightarrow R 5}(t)=1-1,07^{-t-20} ; \gamma_{R 5 \rightarrow R 5 X 4}(t)=1,07^{-t-20} ; \gamma_{R 5 X 4 \rightarrow R 5}(t)=0,02 ; \gamma_{R 5 \times 4 \rightarrow R 5 \times 4}(t)=0,97 ; \gamma_{R 5 \times 4 \rightarrow X 4}(t)=0,01 ;$ $\gamma_{X 4 \rightarrow R 5 X 4}(t)=0,01 ; \gamma_{X 4 \rightarrow X_{4}}(t)=0,99$. Note que no gráfico à direita, no qual um horizonte hipotético de 1000 meses é considerado, (11) é verificada. (Direita) Evolução do fenótipo SI (R5X4+X4) associada aos gráficos superiores. Em preto, perfil SI correspondente à simulação de (61); em vermelho, perfil correspondente a (1) para $\Omega=82,10 \%$ e $\tau=0,46 \%$. Note a ocorrência do pico populacional da linhagem $S I 18$ meses da eclosão da população SI no hospedeiro.

Figure 4 (a) Population dynamics for $R 5, R 5 X 4$ and $X 4$ strains produced by simulation of (3-4) regarding adjusted values of $\gamma_{a \rightarrow b^{\prime}}(a, b) \in E$ for $\Omega$ $=80 \%$; (b) (Left) Simulated profiles for HIV-1 population dynamics after SI phenotype appearance in the host, according to (6). Here, $\gamma_{R 5 \rightarrow R 5}=0.95$; $\gamma_{R 5 \rightarrow R 5 X 4}=0.05 ; \gamma_{R 5 X 4 \rightarrow R 5}=0.02 ; \gamma_{R 5 X 4 \rightarrow R 5 X 4}=0.97 ; \gamma_{R 5 X 4 \rightarrow X 4}=0.01 ; \gamma_{X 4 \rightarrow R 5 X 4}=0.01 ; \gamma_{X 4 \rightarrow X 4}=0.99$. (Right) SI (R5X4+X4) population dynamics. In black: model simulation of eq. (6); in red: model simulation of eq. (1) for $\Omega=83.33 \%$ and $\tau \rightarrow 0 \%$; (c) (Left) $R 5$, R5X4 and X4 strain population dynamics after full-blown SI phenotype according to model simulation of eq. (6) with dynamic mutation rates. Here, $\gamma_{R 5 \rightarrow R 5}(t)=1-1.07^{-t-20} ; \gamma_{R 5 \rightarrow R 5 X 4}(t)=1.07^{-t-20}$; $\gamma_{R 5 X 4 \rightarrow R 5}(t)=0,02 ; \gamma_{R 5 X 4 \rightarrow R 5 X 4}(t)=0.97 ; \gamma_{R 5 X 4 \rightarrow X 4}(t)=0.01 ; \gamma_{X 4 \rightarrow R 5 X 4}(t)=0.01 ; \gamma_{X 4 \rightarrow X 4}(t)=0,99$. In the chart on the right, in which a hypothetical time horizon of 1,000 months is considered, conditions (11) are verified. (Right) SI (R5X4+X4) phenotype evolution associated with the upper charts. In black: SI simulated profile for (6); in red: profile associated with eq. (1) for $\Omega=82.10 \%$ and $\tau=0.46 \%$. The SI population peak occurs 18 months after appearance of SI phenotype in the host. 
Claramente, se $\gamma_{R 5 \rightarrow R 5 X 4}(0)>0$, para $t=0$ tem-se que:

$\frac{d F_{S I}(t)}{d t}>0$

pois $V_{R 5}(0)=1$ e $V_{R 5 X 4}(0)=V_{X 4}(0)=0$ (condições iniciais de 9). Logo, $\exists t>0 \mid F_{S I}(t)>0$ (lembrar que $\left.F_{S I}(t)=V_{R 5 X 4}(t)+V_{X 4}(t)\right)$. Seja $\gamma_{R 5 \rightarrow R 5 X 4}(t)$ uma função assintótica (assíntota $\mathrm{f}(t)=0$ ) decrescente com imagem $\operatorname{Im} \gamma_{R 5 \rightarrow R 5 X 4}(t)$ $\subset$ ]0,1], $\forall t$. A função $\gamma_{R 5 \rightarrow R 5}(t)$ será, como consequência, assintótica (assíntota $\mathrm{f}(t)=1$ ) crescente $\operatorname{Im} \gamma_{R 5 \rightarrow R 5}(t) \subset[0,1[, \forall t$. Para estas funções, (9) torna-se:

$$
\begin{aligned}
& V_{R 5}^{S S}=\underbrace{\frac{\gamma_{R 5 X 4 \rightarrow R 5}(t) \cdot \gamma_{X 4 \rightarrow R 5 X 4}(t)}{\gamma_{R 5 \rightarrow R 5 X 4}(t)} \cdot \gamma_{X 4 \rightarrow R 5 X 4}(t)+\gamma_{R 5 X 4 \rightarrow R 5}(t) \cdot \gamma_{X 4 \rightarrow R 5 X 4}(t)+\gamma_{R 5 X 4 \rightarrow X 4}(t) \cdot \underbrace{\gamma_{R 5 \rightarrow R 5 X 4}(t)}_{=0}}_{=0}=1 \\
& V_{R 5 X 4}^{S S}=\frac{\overbrace{\gamma_{R 5 \rightarrow R 5 X 4}(t)}^{\gamma_{X}} \cdot \gamma_{X 4 \rightarrow R 5 X 4}}{\gamma_{R 5 \rightarrow R 5 X 4}(t) \cdot \gamma_{X 4 \rightarrow R 5 X 4}(t)+\gamma_{R 5 X 4 \rightarrow R 5}(t) \cdot \gamma_{X 4 \rightarrow R 5 X 4}(t)+\gamma_{R 5 X 4 \rightarrow X 4}(t) \cdot \gamma_{R 5 \rightarrow R 5 X 4}(t)}=0 \\
& V_{X 4}^{S S}=\frac{\overbrace{\gamma_{R 5 \rightarrow R 5 X 4}(t) \cdot \gamma_{R 5 X 4 \rightarrow X 4}(t)}^{=0}(t) \cdot \gamma_{X 4 \rightarrow R 5 X 4}(t)+\gamma_{R 5 X 4 \rightarrow X 4}(t) \cdot \gamma_{R 5 \rightarrow R 5 X 4}(t)}{\gamma_{R 5 \rightarrow R 5 X 4}(t) \cdot \gamma_{X 4 \rightarrow R 5 X 4}(t)+\gamma_{R 5 X 4 \rightarrow R 5}(t)}=0
\end{aligned}
$$

ou seja, para $\mathrm{t} \rightarrow \infty$ o sistema retorna à sua condição inicial, isto é, $F_{S I}(0)=F_{S I}\left(t^{s s}\right)=0$. Dado que $\exists t>0 \mid F_{S I}(t)>0$, a ocorrência de (ao menos) um pico populacional de variantes SI é assegurada. Note que, para tanto, a relaxação $\gamma_{a \rightarrow b},(a, b) \in E^{R}$, em $\gamma_{a \rightarrow b}(t),(a, b) \in E^{R}$ pode ser restrita à geração de funções dinâmicas $\gamma_{a \rightarrow b}(t),(a, b) \in E^{R} \mid a=\mathrm{R} 5$, ou seja, os resultados de (11) independem da dinâmica das taxas de mutação $\gamma_{a \rightarrow b}(t),(a, b) \in E^{R} \mid a=\mathrm{R} 5 \mathrm{X} 4, \mathrm{X} 4$.

A Figura 4c apresenta resultados gerados pela relaxação $\gamma_{a \rightarrow b}$ em $\gamma_{a \rightarrow b}(t),(a, b) \in E^{R}$. Tais resultados apresentam importante harmonia em relação à literatura no que se refere à frequente predominância da população R5X4 nas fases finais da infecção ${ }^{20}$ (em geral, subsequente à emergência de variantes SI no hospedeiro).

\section{Conclusões}

Em razão da infecção HIV-1/AIDS não ser ainda compreendida em detalhes, a modelagem matemática da imunologia e patogênese do HIV-1 tem provado não apenas ser uma eficiente ferramenta para a avaliação e entendimento dos mecanismos dinâmicos que governam o curso da infecção, mas também elemento vital para o abandono de paradigmas inicialmente aceitos acerca desta patogênese ${ }^{15}$.
Neste contexto, a identificação de CCR5 e CXCR4 como principais co-receptores para a infecção pelo HIV-1 tem, além de propiciado melhor compreensão do tropismo viral e da progressão da doença, motivado o tratamento matemático da questão ${ }^{16}$. Neste artigo, diferentes modelos de evolução fenotípica do HIV-1 em termos da habilidade retroviral de induzir a formação de sincício são testados e discutidos. A aderência a resultados experimentais é mais bem verificada quando a reversibilidade entre fenótipos SI e NSI é considerada. Em adição, maior alinhamento à dinâmica invivo é observada se as funções dinâmicas no tempo são admitidas para modelar a taxa de mutação entre variantes R5, R5X4 e X4 do HIV-1.

Tal análise matemática do comportamento dinâmico associado ao fenótipo da população viral proporciona elementos adicionais ao entendimento de modelos biológicos anteriores. Viabiliza a proposição de abordagens mais sofisticadas e precisas relacionadas ao controle ótimo da infecção HIV-1/AIDS se considerado que a correlação entre especificidade a diferentes co-receptores e o escape de pressões imunes permanece amplamente inexplorada em termos de políticas práticas relacionadas ao planejamento da quimioterapia anti-HIV-1. 


\section{Referências}

1. Shankarappa R, Margolick JB, Gange SJ, Rodrigo AG, Upchurch D, Farzadegan H et al. Consistent Viral Evolutionary Changes Associated with the Progression of Human Immunodeficiency Virus Type 1 Infection. $J$ Virol 1999; 73(2): 10489-502.

2. Blaak H, Van't Wout AB, Brouwer M, Hooibrink B, Hovenkamp E, Schuitemaker H. In Vivo HIV-1 Infection of CD $45 \mathrm{RA}^{+\mathrm{C}} \mathrm{D} 4^{+} \mathrm{T}$ Cells is Established Primarily by Syncytium-Inducing Variants and Correlates with the Rate of CD4+ T Cell Decline. PNAS-USA 2000; 97(3): 1269-74.

3. Shieh B, Liau Y, Hsieh P, Yan Y, Wang S, Li C. Influence of Nucleotide Polymorphisms in the CCR2 Gene and the CCR5 Promoter on the Expression of Cell Surface CCR5 and CXCR4. Int Immunol 2000; 12(9): 1311-8.

4. YiY, Isaacs SN, Williams DA, Frank I, Schols D, De Clercq E et al. Role of CXCR4 in Cell-Cell Fusion and Infection of Monocyte-Derived Macrophages by Primary Human Immunodeficiency Virus Type 1 (HIV-1) Strains: Two Distinct Mechanisms of HIV-1 Dual Tropism. J Virol 1999; 73(9): 7117-25.

5. Stilianakis NI, Boucher CAB, Jong MD, Leeuwen RV, Schuurman R, Boer RJ. Clinical Data Sets of Human Immunodeficiency Virus Type 1 Reverse TranscriptaseResistant Mutants Explained by a Mathematical Model. $J$ Virol 1997; 71(1): 161-8.

6. Yamaguchi Y, Gojobori T. Evolutionary Mechanisms and Population Dynamics of the Third Variable Envelope Region of HIV within Single Hosts. PNAS-USA 1997; 94: 1264-9.

7. Connor RI, Sheridan KE, Ceradini D, Choe S, Landau NR. Change in Coreceptor Use Correlates with Disease Progression in HIV-1-Infected Individuals. J Exp Med 1997; 185(4): 621-8.

8. Hu Q, Barry AP, Wang Z, Connolly SM, Peiper SC, Greenberg ML. Evolution of the Human Immunodeficiency Virus Type 1 Envelope during Infection Reveals Molecular Corollaries of Specificity for Coreceptor Utilization and AIDS Pathogenesis. J Virol 2000; 74(24): 11858-72.

9. Van't Wout AB, Ran LJ, Kuiken CL, Kooststra NA, Pals ST, Schuitemaker H. Analysis of the Temporal Relationship between Human Immunodeficiency Virus Type 1 Quasispecies in Sequential Blood Samples and Various Organs Obtained at Autopsy. J Virol 1998; 72(1): 488-96.

10. Bagnarelli P, Mazzola F, Menzo S, Montroni M, Butini L, Clementi M. Host-Specific Modulation of the Selective Constraints Driving Human Immunodeficiency Virus Type 1 Env Gene Evolution. J Virol 1999; 73(5): 3764-77.
11. Cornelissen M, Burg R, Zorgdrager F, Lukashov V, Goudsmit J. Gene Diversity of Five Human Immunodeficiency Virus Type 1 Subtypes: Evidence for Naturally Occurring Mutations That Contribute to Drug Resistance, Limited Recombination Patterns, and Common Ancestry for Subtypes B and D. J Virol 1997; 71(9): 6348-58.

12. Fields BN, Knipe DM, Howley PM. (editors-in-chief). Fundamental Virology. Philadelphia (PA): $3^{\text {a }}$ ed. Lippincott-Raven Publishers; 1996.

13. Bleul, C.C.; Wu, L.; Hoxie, J.A.; Springer, T.A.; Mackay, C.R. The HIV Coreceptors CXCR4 and CCR5 are Differentially Expressed and Regulated on Human T Lymphocytes. PNAS USA 1997, 94, 1925-1930.

14. Philpot S, Weiser B, Anastos K, Kitchen CMR, Robison E, Meyer III et al. Preferential Suppression of CXCR4Specific Strains of HIV-1 by Antiviral Therapy. J Clin Invest 2001; 107(4): 431-8.

15. Wei X, Ghosh SK, Taylor ME, Johnson VA, Emini EA, Deutsch P et al. Viral Dynamics in Human Immunodeficiency Virus Type 1 Infection. Nature 1995; 373(12): 117-22.

16. Joly M. Pinto JM. The Role of Mathematical Modeling on the Optimal Control of HIV-1 Pathogenesis. AIChEJ 2006; 52(3): 1-28.

17. Perelson AS, Neumann AU, Markowitz M, Leonard JM, Ho DD. HIV-1 Dynamics in Vivo: Virion Clearance Rate, Infected Cell Life-Span, and Viral Generation Time. Science 1996; 271(15): 1582-6.

18. Brooke A, Kendrick D, Meeraus A, Raman R. GAMS - A user's guide. GAMS Development Corp.: Washington, DC; 1998.

19. Drud AS. A system for large scale nonlinear optimization, reference manual for Conopt subroutine library. Bagsvaerd (Dinamarca): ARKI Cons. \& Develop. A/S; 1996.

20. Singh A, Collman RG. Heterogeneous Spectrum of Coreceptor Usage among Variants within a Dualtropic Human Immunodeficiency Virus Type 1 Primary-Isolate Quasispecies. JVirol 2000; 74(21): 10229-35.

Recebido em: 18/08/09 Versão final reapresentada em: 08/03/10 Aprovado em: 30/03/10 